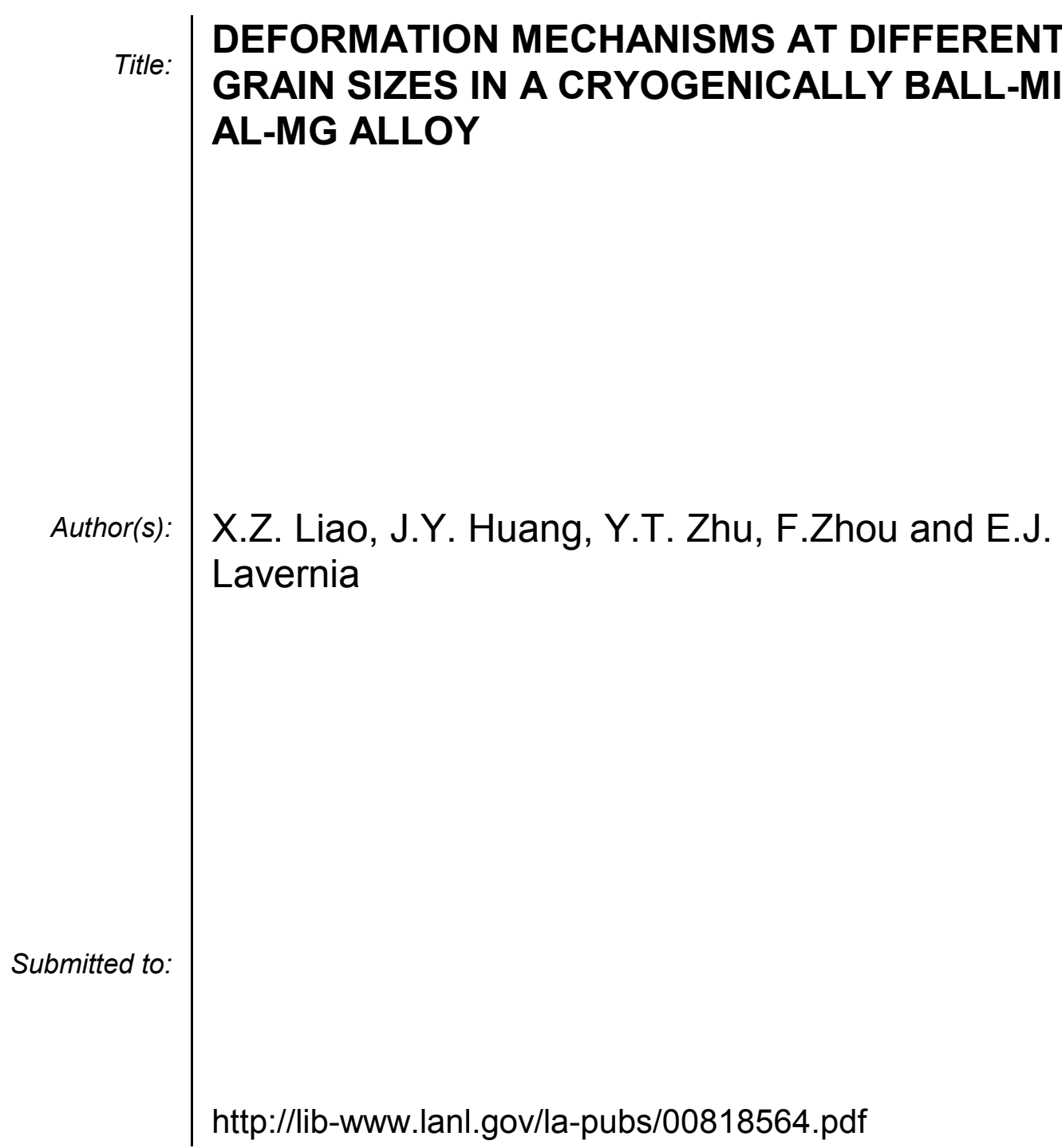

Los Alamos National Laboratory, an affirmative action/equal opportunity employer, is operated by the University of California for the U.S. Department of Energy under contract W-7405-ENG-36. By acceptance of this article, the publisher recognizes that the U.S. Government retains a nonexclusive, royaltyfree license to publish or reproduce the published form of this contribution, or to allow others to do so, for U.S. Government purposes. Los Alamos National Laboratory requests that the publisher identify this article as work performed under the auspices of the U.S. Department of Energy. Los Alamos National Laboratory strongly supports academic freedom and a researcher's right to publish; as an institution, however, the Laboratory does not endorse the viewpoint of a publication or guarantee its technical correctness. 


\title{
DEFORMATION MECHANISMS AT DIFFERENT GRAIN SIZES IN A CRYOGENICALLY BALL-MILLED AL-MG ALLOY
}

\author{
X.Z. Liao ${ }^{1}$, J.Y. Huang ${ }^{1}$, Y.T. Zhu ${ }^{1}$, F.Zhou ${ }^{2}$ and E.J. Lavernia ${ }^{2}$ \\ ${ }^{1}$ Division of Materials Science and Technology, Los Alamos National Laboratory \\ Los Alamos, NM 87545 \\ ${ }^{2}$ Department of Chemical and Biochemical Engineering and Materials Science \\ University of California, Irvine, CA 92697
}

\begin{abstract}
An Al-7.5 wt. \% Mg alloy was ball-milled in liquid $\mathrm{N}_{2}$ for eight hours and its microstructures were investigated using transmission electron microscopy. Electron diffraction confirmed that the resulting powder is a supersaturated Al-Mg solid solution with a face-centered cubic structure. Three nanostructures with different grain size ranges and shapes were observed and the deformation mechanisms in these structures were found to be different. The reasons for the different deformation mechanisms were discussed.
\end{abstract}

Keywords: Aluminum alloy; Cryogenic ball milling; Transmission electron microscopy; Microstructure.

\section{Introduction}

Mechanical ball milling (BM) is one of the severe plastic deformation (SPD) techniques that generate nanostructures by plastically deforming the coarse-grained materials. Although BM has been widely used for the preparation of nano-structured materials in recent years [1], the structural evolution and deformation mechanisms in materials during the BM process have not been well studied.

Two deformation mechanisms have been proposed to be responsible for the structural evolution during BM [2]. One is based on the dislocation accumulation and the other is based on grain rotation and grain boundary sliding [3]. These two deformation mechanisms were supposed to happen in materials ball-milled at ambient temperature. BM under cryogenic conditions may greatly affect the defect behaviors in the powder crystallites and therefore will certainly result in change in deformation mechanisms. However, no detailed investigation on the microstructures and deformation mechanisms in cryogenic BM materials has yet been reported. In this paper, we use high-resolution transmission electron microscopy (HREM) to investigate the microstructures of Al-7.5 wt.\% Mg alloy powder obtained from cryogenic BM and report three deformation mechanisms in grains with three different size ranges, respectively.

\section{Experimental procedures}

Spray-atomized $\mathrm{Al}-7.5$ wt.\% $\mathrm{Mg}(\mathrm{Al}-8.3$ at.\% $\mathrm{Mg}$ ) alloy powder with particle sizes in the range of 10 to $40 \mu \mathrm{m}$ were used as starting materials for cryogenic BM. The milling was carried out in a modified Union Process 01-HD attritor with a stainless steel 
vial and balls $(6.4 \mathrm{~mm}$ in diameter) at a rate of $180 \mathrm{rpm}$ for 8 hours. The ball-to-powder weight ratio was $36: 1$. During the milling operation, liquid nitrogen was added into the mill to maintain complete immersion of the milling media, which keeps the temperature at $-190^{\circ} \mathrm{C}$. Prior to milling, about 0.25 wt.\% of stearic acid $\left(\mathrm{CH}_{3}\left(\mathrm{CH}_{2}\right)_{16} \mathrm{CO}_{2} \mathrm{H}\right)$ was added to the powders as a process control agent to moderate the cold welding process.

The resulting powder was pressed under a pressure of $1.1 \mathrm{GPa}$ to form a small pellet, which was then mechanically ground to a thickness of about $50 \mu \mathrm{m}$. Further thinning to a thickness of electron transparency was carried out using Gatan precision ion polishing system with $\mathrm{Ar}^{+}$accelerating voltage of $3.5 \mathrm{kV}$. HREM investigation was carried out using a JEOL $3000 \mathrm{~F}$ transmission electron microscope (TEM) working at 300 $\mathrm{kV}$.

\section{Results and Discussion}

TEM investigation shows that the as-milled powder was not deformed homogenously and different stages of the deformation process co-existed during the cryogenic BM. Three kinds of nanostructures with different grain size ranges have been found in the sample. Figure 1(a) shows a typical image of the largest crystallites having a lamellate structure with a length range of $100-200 \mathrm{~nm}$ and a width of around $30 \mathrm{~nm}$. Figure 1(b) shows a typical structure of the most commonly seen equiaxed crystallites having diameters of around $10-30 \mathrm{~nm}$ and Fig. 1(c) presents the smallest crystallites with a dimension of less then $10 \mathrm{~nm}$. As will be discussed later, these three kinds of structures were produced by three different deformation mechanisms.

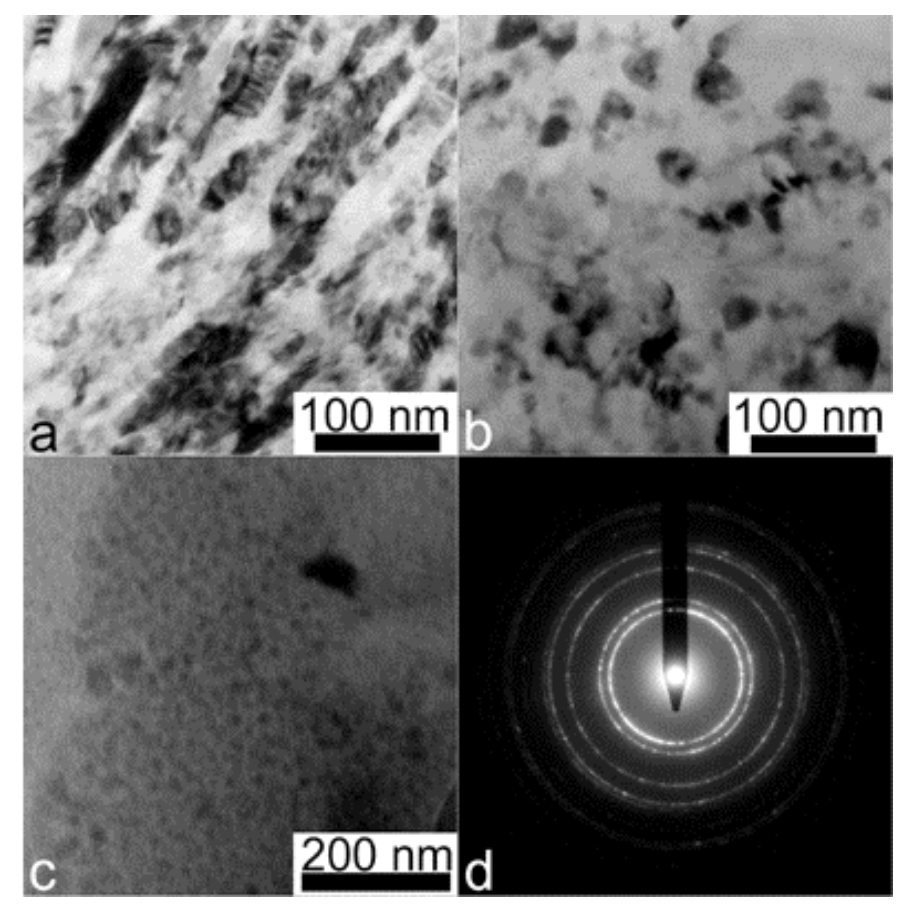

Figure 1 Nanostructures of three different sizes of crystallites. (a) Large crystallites; (b) medium-sized crystallites; (c) the smallest crystallites; (d) an electron diffraction pattern taken from the area shown in (a). 
Electron diffraction patterns taken from the above three typical areas indicate that they are all supersaturated Al-Mg solid solution with a fcc structure. An example of the electron diffraction patterns taken from the area shown in Fig. 1(a) is demonstrated in Fig. 1(d). Although the equilibrium solubility of $\mathrm{Mg}$ in $\mathrm{Al}$ at room temperature is only 1.2 at.\%, much lower than the 8.3 at.\% observed in this investigation, metastable supersaturated $\mathrm{Al}(\mathrm{Mg})$ fcc solid solutions with very high $\mathrm{Mg}$ contents have been reported in mechanically alloyed samples. For example, the solubility of $\mathrm{Mg}$ in $\mathrm{Al}$ has been reported up to 18 at. $\%$ in $\mathrm{Al}_{70} \mathrm{Mg}_{30}$ [4], 23 at.\% in $\mathrm{Al}_{60} \mathrm{Mg}_{40}$ [5], and 45 at.\% in $\mathrm{Al}_{50} \mathrm{Mg}_{50}$ [4].

HREM imaging has been carried out to understand how the crystallites with different sizes deform. Figure 2(a) is an HREM image of a large crystallite taken along a $<110>$. A very high density of dislocations is found in the crystallites. Most of the dislocations are $60^{\circ}$ dislocations with Burgers vectors of $1 / 2<110>$. To see the dislocation array and the dislocation density clearly, the area marked with a black square in Fig. 2(a) was Fourier-transformed and then inverse-transformed using only (000) and a pair of $\{111\}$. The resulting image with a higher magnification is shown in Fig. 2(b), in which only a group of $\{111\}$ planes are seen. The dislocation density measured from Fig. 2(b) is $1.3 \times 10^{17} / \mathrm{m}^{2}$. The real dislocation density is expected to be higher when dislocations on other $\{111\}$ are included. It is clear from Fig. 2(b) that many dislocations appear as dipoles, which have been presented in many heavily deformed metals. The dipoles are in fact dislocation loops with elongation along one direction so that they look like a pair of single dislocations with opposite Burgers vectors. The formation mechanism of these elongated loops in fcc metals has been discussed by Fourie and Murphy [6].

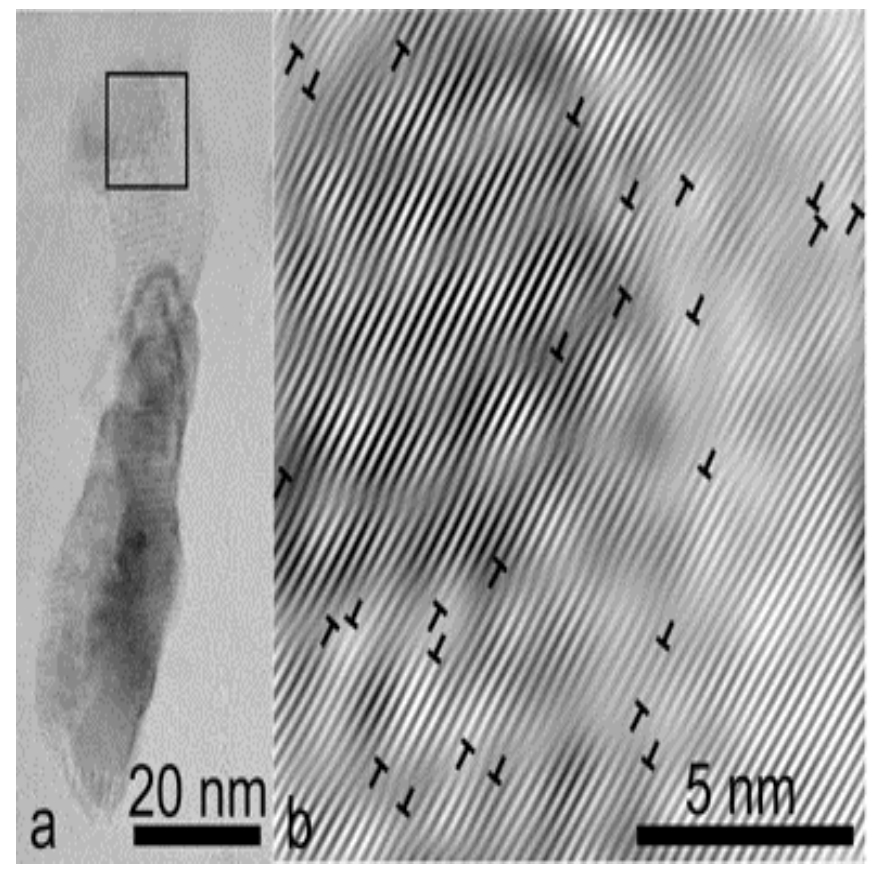

Figure 2 (a) A typical HREM image of a large crystallite; (b) an enlarged Fourier-filtered image of the area marked with a black square in (a) imaged by using (000) and a pair of $\{111\}$. Dislocations shown in (b) are indicated with black "T". 
Large crystallites transformed into medium-sized crystallites through the formation of small-angle grain boundaries (SAGBs). Figure 3 shows a typical example of SAGBs where misorientations between neighboring medium-sized crystallites are seen. The SAGBs in Fig.3 are marked with black stars. Three black straight lines were drawn parallel to the $\{111\}$ planes in neighboring crystallites $\mathrm{A}, \mathrm{B}$ and $\mathrm{C}$, respectively. Misorientations of about $3^{\circ}$ between the neighboring crystallites are clearly revealed. We believe that the randomly distributed dislocations in large crystallites first interacted and rearranged to form a string of dislocation cells along the longitudinal axis of the elongated large crystallites. These cell boundaries then transformed to SAGBs with increasing deformation. Dislocations are rarely seen within these medium-sized crystallites (a dislocation in crystallite D is seen and marked), indicating that SAGBs acted both as dislocation sources and as dislocation sinks during the deformation. The fact that two-dimensional lattice fringes are seen in crystallites A and $\mathrm{C}$ while only onedimensional lattice fringes are seen in $\mathrm{B}$ implies the rotation of $\mathrm{B}$ relative to $\mathrm{A}$ and $\mathrm{C}$. Note that crystallite D has twin relationship with $\mathrm{C}$ and $\mathrm{B}$ and this is marked in Fig.3.

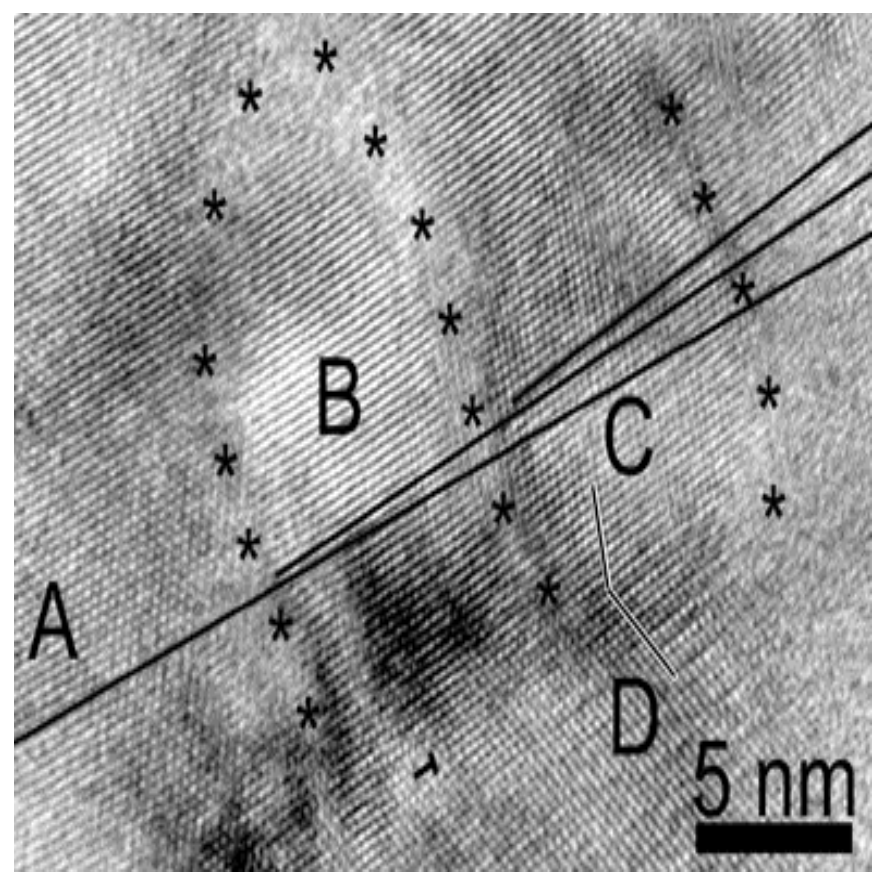

Figure 3 An HREM image of medium-sized crystallites. SAGBs are marked with black stars and the small tilting angles among crystallites A, B and C are presented with black straight lines. Crystallite D has a twin relationship with $\mathrm{B}$ and $\mathrm{C}$. The relationship is indicated by marking the $\{111\}$ at either side of the twin boundary. A dislocation in D is indicated with a black "T".

While twins are seen only occasionally in medium-sized crystallites, they are more frequently observed in crystallites with dimensions smaller than $10 \mathrm{~nm}$. A typical example is shown in Fig. 4, where crystallite $\mathrm{A}$ is a five-fold twin while micro-twins and stacking faults exist in crystallite $\mathrm{B}$. Crystallite $\mathrm{C}$ has a $5^{\circ}$ misorientation with crystallite A. The position of the boundary between $\mathrm{A}$ and $\mathrm{C}$ is marked with two arrows. No dislocation is seen at the boundary and the small-angle misorientation between $\mathrm{A}$ and $\mathrm{C}$ is accommodated by the lattice strain in the two neighboring crystallites. 
Figure 5 shows another typical example of a five-fold twin, which has a dimension of about $3 \mathrm{~nm}$. Because of extremely large lattice strain, severe lattice distortion is seen in the five-fold twin. However, the twin boundaries can still be identified and are indicated with arrows. Each twin domain is marked with number 1 to 5, respectively. Crystallites surrounding the twins are marked with $\mathrm{A}, \mathrm{B}, \mathrm{C}$ and $\mathrm{D}$. Small angle misorientations between 1 and $\mathrm{A}, 2$ and $\mathrm{B}, 4$ and $\mathrm{C}$, and 5 and $\mathrm{D}$ are seen in Fig. 5. These misorientations imply (i) A, B, C, and D had twin relationships which were later destroyed by further deformation and (ii) the five-fold twin was the center of the twins A, B, C and D. The relationships among the crystallites in Fig. 5 strongly imply that the five-fold twin is formed through severe mechanical deformation, not re-crystallization of the sample.

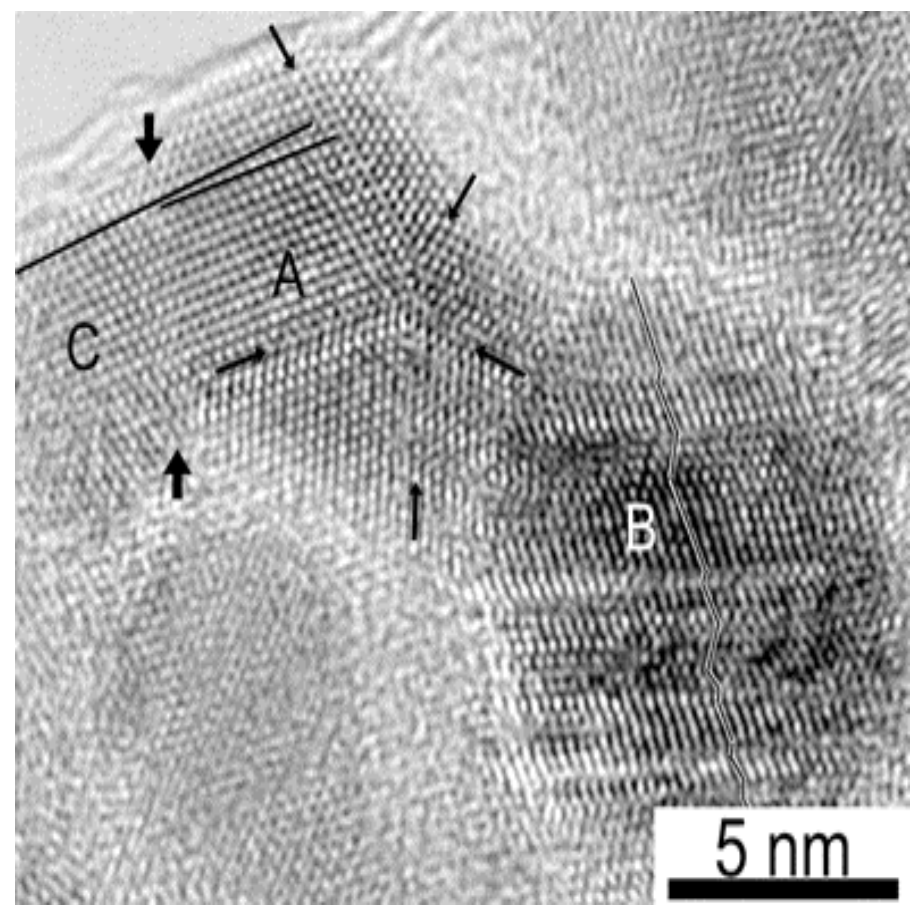

Figure 4 A typical HREM image of crystallites with dimensions smaller than $10 \mathrm{~nm}$. Crystallite A is a five-fold twin. Micro-twins and stacking faults are seen in crystallite B. There is a $5^{\circ}$ misorientation between $\mathrm{A}$ and $\mathrm{C}$. The SAGB between $\mathrm{A}$ and $\mathrm{C}$ is marked with two large black arrows.

Twinning and slip are two major competitive mechanisms of plastic deformation. Grain size plays an important role in determining the deformation mechanism at different deformation stages. According to Venables [7], the stress required to activate a twinning dislocation of length $l$ is

$$
\tau_{T}=S / b_{1}+G b_{1} / l
$$

where $S$ is the stacking fault energy (SFE), $b_{1}=1 / 6<112>$ is the Burgers vector of the Shockley partial dislocation involved in twinning, and $\mathrm{G}$ is the shear modulus. Johari and Thomas [8] suggested that with increasing plastic deformation the dislocation density increases to a value at which the critical length of the pinned dislocation $u$ is attained. The shear stress to bow out such a dislocation is given by 


$$
\tau_{D}=G b / u
$$

where $b=1 / 2<110>$ is the Burgers vector of the unit dislocation.

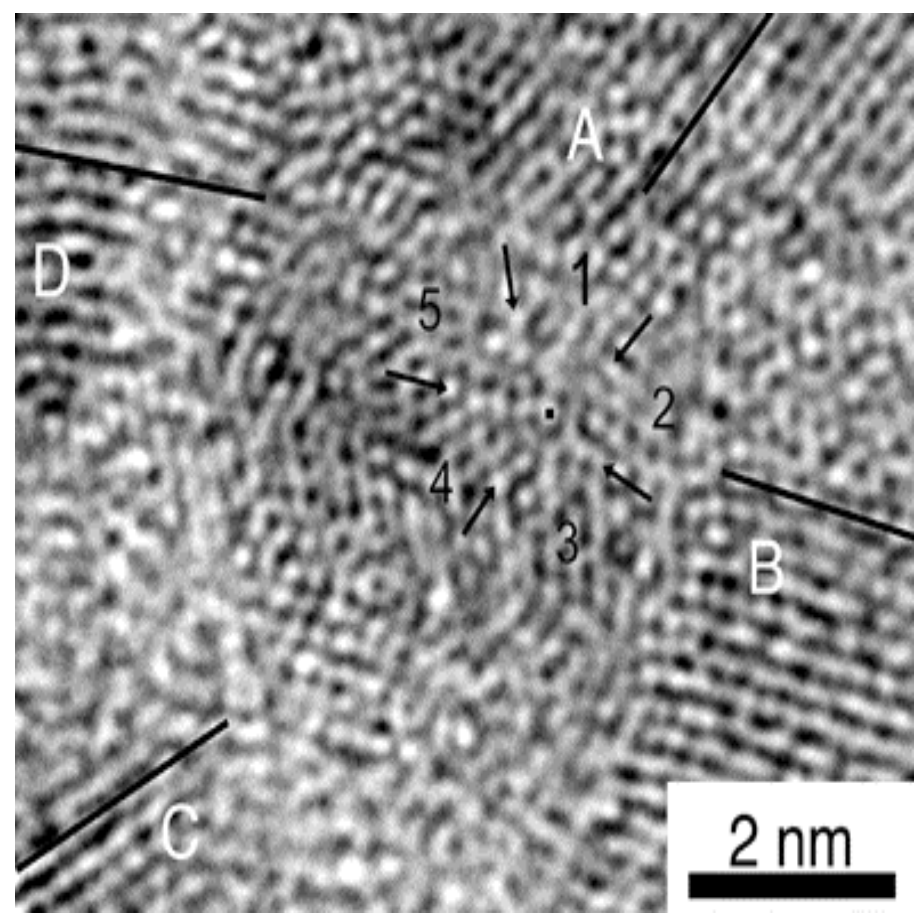

Figure 5 Another example of a five-fold twin. The twin boundaries are marked with small arrows and each twin domain is labeled with $1,2,3,4$, and 5, respectively. The twin center is highlighted with a black square dot. A group of $\{111\}$ planes in crystallites A, B, C, and D surrounding the twin is indicated with black straight lines.

By reasonably assuming both $l$ and $u$ are equal to the crystallite grain size $\mathrm{d}$, we will see from equations (1) and (2) that when the grain size $\mathrm{d}$ is large, $\tau_{T}>\tau_{D}$, especially for large SFE materials such as $\mathrm{Al}$ and $\mathrm{Al}$-alloys (the SFEs of pure $\mathrm{Al}$ and $\mathrm{Al}-3 \% \mathrm{Mg}$ are $166 \mathrm{~mJ} / \mathrm{m} 2$ [9] and $190 \mathrm{~mJ} / \mathrm{m} 2$ [10], respectively). In this case, slip is the favorable deformation mechanism. This explains the fact that no twin is seen in large grains.

During the slip deformation process, the density of dislocations increases resulting in an increase in the lattice strain. The increased lattice strain at the early stage of BM has been confirmed by X-ray diffraction data [11]. Further deformation results in dislocation rearrangement, forming SAGBs and medium-sized crystallites. The interaction of dislocations to form SAGBs greatly reduces dislocation density within the medium-sized crystallites, as evidenced in Fig. 3, and consequently the lattice strain. The reduction of lattice micro-strain at this stage has also been confirmed by X-ray diffraction data [11]. Twins are observed occasionally in medium-sized crystallites. Figure 8 shows an example of a twin seen at this stage.

The SAGBs were later transformed to LAGBs with increasing deformation. This is evidenced in Fig. 1(b) in which most of the grain boundaries among medium-sized 
crystallites are LAGBs. These LAGBs can be achieved primarily through dislocation slip. Grain rotation and grain boundary sliding may also become active after the formation of LAGBs. The in situ TEM observation of deformation behavior of nanometer-sized gold thin films confirmed that grain rotation and grain boundary sliding play a significant role in a plastic deformation process of the nanostructured material [3].

Because $b$ in equation (2) is $\sqrt{3}$ times larger than $b_{1}$ in equation (1), which means the slope of $\tau-1 / \mathrm{d}$ curve is steeper for $\tau_{D}, \tau_{D}$ increases faster than $\tau_{T}$ with decreasing grain size. At a critical small grain size below which we will have $\tau_{T}<\tau_{D}$, twining becomes a favorable deformation mechanism.

It is well known that the SPD produces non-uniform microstructures. Specifically, the grain size distribution in material processed by SPD usually follows a lognormal statistical function [12]. Such a nonuniform structure is also obvious in the cryogenically ball-milled Al-alloy studied here. As shown in Fig. 1, the largest grains are $30 \mathrm{~nm}$ wide and $200 \mathrm{~nm}$ long, while the smallest grains are less than $10 \mathrm{~nm}$ in diameter.

Low temperature also promotes twinning because a twinning stress vs temperature curve increases less steeply at low temperature than the yield stress or the flow stress for plastic deformation by slip [13]. High strain rate has the same effect as low temperature has on twinning [13]. Therefore, cryogenic BM favors twinning more than many other SPD techniques that operate at higher temperatures and lower strain rates.

Deformation twins were observed in $\mathrm{Al}-4.8 \mathrm{wt} \% \mathrm{Mg}$ subjected to shock loading at $-180^{\circ} \mathrm{C}$ by Gray while they were not seen in pure Al under the same deformation conditions [14]. Gray claimed that adding $\mathrm{Mg}$ solute in Al results in increased lattice frictional stress, enhanced rate of work hardening, and reduction in the dynamic recovery rate. These features would, in turn, radically alter the dislocation substructure, resulting in changes in the internal stress pattern. His arguments were consistent with experiments on body-centered cubic lattices where twin formation is favored by solid solution alloying [15] and is attributed to solid solution rather than intrinsic SFE effects [16]. X-ray diffraction investigation of the cryogenic BM Al-7.5 $\mathrm{wt} \% \mathrm{Mg}$ [11] shows the shape of fcc Al peaks changes from asymmetric, which suggests inhomogeneity of $\mathrm{Mg}$ distribution in fcc Al lattice at early stages of deformation, to symmetric, indicating uniform Al-Mg solid solution after long time milling. These results imply that $\mathrm{Mg}$ solute might also play a role in the twinning deformation shown in this investigation.

Besides mechanical twining, it is believed that grain rotation and grain boundary sliding play a more important role in the deformation of very small grains than in medium-sized grains as evidenced by the experiment carried out Ke et. al. [3].

\section{Conclusion}

Cryogenic $\mathrm{BM}$ of $\mathrm{Al}-7.5$ wt.\% $\mathrm{Mg}$ powder was investigated by HREM. The powder is not deformed uniformly and three nanostructures with different grain sizes and shapes were observed co-existing in the powder. At large grain sizes, powder grains are of a lamellar structure with a length range of $100-200 \mathrm{~nm}$ and a width of around $30 \mathrm{~nm}$. 
Deformation in these large grains is mainly based on dislocation slip and the dislocation densities within the large grains are very high. At medium grain sizes, equiaxed grains are in the size range of $10-30 \mathrm{~nm}$. Grain size reduction from large grains to mediumsized grains is achieved through dislocation rearrangement forming SAGBs. The dislocation rearrangement largely reduces the amount of dislocations within mediumsized grains. The SAGBs later transform to LAGBs through dislocation slip. Due to the small grain size, cryogenic condition, relatively high strain rate and $\mathrm{Mg}$ solute effects, twining becomes an important deformation mechanism at grain size smaller than $10 \mathrm{~nm}$.

\section{Acknowledgments}

This project is supported by the Department of Energy NIS-IPP program and the Office of Naval Research (grants N00014-00-1-0109 and N00014-01-1-0882).

\section{References}

1 C. C. Koch, "Synthesis of Nanostructured Materials by Mechanical Milling: Problems and Opportunities", Nanostructured Mater., 9 (1997) 13- 22.

2 E. Hellstern, H. J. Fecht, , Z. Fuand W. L. Johnson, "Structural and Thermodynamic Properties of Heavily Mechanically Deformed Ru and AlRu”, J. Appl. Phys., 65 (1989) 305 - 310.

3 M. Ke, S. A.Hackney, W. W. Milligan, and E. C. Aifantis, "Observation and Measurement of Grain Rotation and Plastic Strain in Nanostructured Metal Thin-Films", Nanostructured Mater., 5 (1995) 689 - 697.

4 A. Calka, W. Kaczmarek, and J. S. Williams, "Extended Solid Solubility in Ball-Milled Al-Mg Alloys", J. Mater. Sci., 28(1993) 15 - 18.

5 D. L. Zhang, T. B. Massalski, and M. R. Paruchuri, "Formation of Metastable and Equilibrium Phases During Mechanical Alloying of Al and Mg Powders", Metall. Mater. Trans. A, 25 (1994) $73-79$.

6 J. T. Fourie and R. J. Murphy, Phil. Mag., 7 (1962) 1617.

7 J. A. Venables, Phil. Mag., 6 (1961) 379.

8 O. Johari and G. Thomas, Acta Metall., 2 (1964) 1152.

9 D. L. Medlin, M. J. Mills, W. M. Stobbs, M. S. Daw, and F. Cosandey, Materials Research Society Symposium Proceedings, 295 (1993) 91

10 F. A. Mohamed, and T. G. Langdon, "Transition from Dislocation Climb to Viscous Glide in Creep of Solid-Solution Alloys", Acta Metall., 22 (1974) 779 - 788.

11 F. Zhou, R. Rodriguez, and E. J. Lavernia, J. Met. Nano. Mater., 2001 (in press).

12 I. V.Alexandrov, Y. T. Zhu, T. C. Lowe, R. K. Islamgaliev, and R. Z. Valiev, "Microstructures and Properties of Nanocomposites Obtained Through SPTS Consolidation of Powders", Metall. Mater. Trans. A, 29 (1998) 2253 - 2260.

13 J. W. Christian and S. Mahajan, "Deformation Twinning", Prog. Mater. Sci., 39 (1995) 1 157.

14 Gray, G. T. III, "Deformation Twinning in Al-4.8 Wt.\% Mg”, Acta Metall., 36 (1988) 1745 1754.

15 S. Mahajan and D. F. Williams, Int. Metall. Rev., 18 (1973) 43.

16 J. O. Stiegler and C. J. McHargue, in Deformation Twinning (edited by R. E. Reed-Hill, J. P. Hirth and H. C. Rogers), p. 209. Gorden \& Breach, New York (1964). 